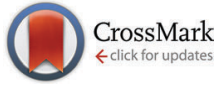

Cite this: Phys. Chem. Chem. Phys., $2016,18,19318$

Received 11th April 2016, Accepted 21st June 2016

DOI: $10.1039 / c 6 c p 02399 a$

www.rsc.org/pccp

\title{
Ice nucleation of an insect lipoprotein ice nucleator (LPIN) correlates with retardation of the hydrogen bond dynamics at the myo-inositol ring
}

\begin{abstract}
Alexander Bäumer, ${ }^{a}$ John G. Duman ${ }^{b}$ and Martina Havenith ${ }^{\star a}$
Remarkably little is known about the mechanism of action of ice nucleation proteins (INPs), although their ability to trigger ice nucleation could be used in a broad variety of applications. We present CD measurements of an insect lipoprotein ice nucleator (LPIN) which show that the lipoproteins consist of a high amount of $\beta$-structures (35\%). Terahertz absorption spectroscopy is used to probe the influence of the LPIN on the H-bond network dynamics. We observe a small, but significant $\mathrm{THz}$ excess, as an indication of an influence on the $\mathrm{H}$-bond network dynamics. When adding the ice nucleation inhibitor sodium borate, this effect is considerably reduced, similar to that observed before for antifreeze glycoproteins (AFGPs). We propose that myo-inositol, the functional group of phosphatidylinositols, is crucial for the observed change of the $\mathrm{H}$-bond network dynamics of hydration water. This hypothesis is confirmed by additional $\mathrm{THz}$ experiments which revealed that the influence of myo-inositol on the hydrogen bond network can be blocked by sodium borate, similar to the case of LPINs. Interestingly, we find a less significant effect when myo-inositol is replaced for chiro- and allo-inositol which underlines the importance of the exact positioning of the $\mathrm{OH}$ groups for the interaction with the $\mathrm{H}$-bond network. We propose that a local ordering of water molecules is supporting ice nucleation activity for the LPIN in a similar way to that found for AFP activity in the case of hyperactive insect AFPs.
\end{abstract}

\section{Introduction}

Freeze tolerance (the ability to survive ice formation in the body fluids, usually only extracellular) is one of nature's strategies for life under sub-zero conditions. This is often achieved by employing extracellular ice nucleation proteins (INPs) which trigger ice nucleation at high sub-zero temperatures, i.e. temperatures just below $0{ }^{\circ} \mathrm{C}$, thereby inhibiting extensive supercooling that can lead to lethal intracellular ice if freezing eventually occurs. ${ }^{1-3}$ Although the freezing point of pure water is $0{ }^{\circ} \mathrm{C}$, water can be supercooled to $-18{ }^{\circ} \mathrm{C}$, where the crystallization rate reaches a maximum and homogeneous nucleation occurs. ${ }^{4}$ Several organisms have developed strategies to promote heterogeneous nucleation at higher sub-zero temperatures, to limit supercooling and promote slower and more controlled freezing, among them are many insects. In general INPs trigger the extracellular formation of ice, resulting in an increased solute concentration in the unfrozen extracellular water, which leads to dehydration of the cells due to osmotic pressure differences. High concentrations of small carbohydrates and glycerol serve as

\footnotetext{
${ }^{a}$ Lehrstuhl für Physikalische Chemie II, Ruhr-Universität, 44801 Bochum, Germany. E-mail: martina.havenith@rub.de

${ }^{b}$ Department of Biological Sciences, University of Notre Dame, Notre Dame, IN 46556, USA
}

intracellular cryoprotective agents, whereas the cell membrane inhibits the propagation of ice into the cell. ${ }^{2,3}$

INPs are known for their broad variety of applications in medicine, industry and especially in agriculture. The frost damage caused by ice nucleating bacteria is responsible for massive crop failure. Despite the commercial interest, surprisingly little is known about the molecular mechanism. In particular no three-dimensional structures from X-ray diffraction or nuclear magnetic resonance spectroscopy are available.

Nevertheless, some INPs have been characterized relatively well, among them is the lipoprotein ice nucleator (LPIN) which is found in the hemolymph of the freeze tolerant larvae of the north American crane fly Tipula trivittata. ${ }^{5-8}$ Similar to other insect hemolymph lipoproteins the LPIN serves as a lipid shuttle, however, the LPIN also shows ice nucleation activity at temperatures of about -6.0 to $-9.5{ }^{\circ} \mathrm{C} .{ }^{6}$ Unlike other lipoproteins, the LPIN contains surface located phosphatidylinositol (PI) molecules. The $\mathrm{OH}$ groups of the myo-inositol ring along with the two apolipoproteins are required for ice nucleation activity. ${ }^{6}$ It is assumed that the two apolipoproteins bind to PI, providing an ice lattice template which forms hydrogen bonds to water and thereby forming and stabilizing ice embryo crystals. ${ }^{6}$ The specific arrangement of the $\mathrm{OH}$ groups at the myo-inositol head of the PIs is proposed to be crucial, thus a substitution by another inositol stereoisomer (e.g. chiro-inositol) 
is expected to result in an inactivation, consequently these stereoisomers are not found in any ice nucleation protein. The ice nucleation activity is dramatically reduced by adding sodium borate, probably by forming complexes with the $\mathrm{OH}$ groups. ${ }^{6}$ Interestingly, this is similar to the case of antifreeze glycoproteins (AFGPs) where complexation of the carbohydrate cis-OH groups by borate was found to suppress the long-range hydration shell - as detected by terahertz absorption - and to strongly reduce the AFGP antifreeze activity. ${ }^{9}$

Most studies of ice nucleating proteins so far focused on bacterial INPs, especially highly potent INPs expressed by Pseudomonas syringae. ${ }^{10-14}$ In particular with the help of computational modeling, some ideas regarding how INPs structure water into ice have been proposed.

It is widely accepted that antifreeze proteins (AFPs) use the adsorption-inhibition mechanism for antifreeze activity. ${ }^{15}$ Although INPs do not use this mechanism of action, they serve opposite biological functions and are in general much larger, and recent studies indicate that both protein classes seem to utilize the same ice-binding mechanism. Garnham et al. performed molecular dynamics (MD) simulations and found that bacterial INPs could order water molecules into an ice-like lattice by an anchored clathrate water mechanism similar to AFPs. ${ }^{16,17}$ Previously, it has been reported that INPs from different bacteria share a typical iceinteracting secondary structure motif with insect AFPs, a $\beta$-helical fold. ${ }^{12,16,18,19}$ Consequently, Graether et al. suggested that the distinguishing feature between INPs and AFPs could be the size of the ice-interacting surface. ${ }^{12}$

However, the underlying molecular mechanism for INPs is not understood. The tertiary structures of INPs have not been solved and thus the mechanism that is responsible for the phase transition at ice nucleation sites of INPs has not yet been identified.

Terahertz (THz) spectroscopy is a valuable tool to study the fast hydration dynamics of water and its hydrogen bond network in the presence of biomolecules. ${ }^{20-22}$ Using concentration dependent THz absorption spectroscopy, we have investigated the influence of AFPs on the collective hydrogen bond dynamics. ${ }^{9,23-25}$ In these cases, a retardation of the collective H-bond network dynamics was observed which is correlated with an increase in THz absorption in the frequency range between 2 and 3 THz. In particular, for AFPs a retardation of hydration dynamics has been found to be a key property for antifreeze activity. ${ }^{9,25-27}$ In recent studies we proposed a two-tier mechanism of action for molecular recognition. ${ }^{28}$ In addition to the enthalpic hydrogen-binding mechanism by $\mathrm{OH}$ groups we found that all major AFP classes showed a retardation of the hydrogen bond dynamics at the ice-binding site. ${ }^{9,25-27}$ The formation of local hydrogen bonds is found to be a necessary condition for antifreeze activity and contributes to the enthalpic component. We proposed that a retardation of the hydrogen bond dynamics at the ice-binding site is favorable for the binding of nano-ice crystals, since it reduces the entropic cost associated with desolvation of the binding site. ${ }^{29}$ The proposal of a retardation of hydrogen bond dynamics at the ice-binding site of AFP III was confirmed by IR SFG measurements by the Bakker group which showed an ice-like water layer at the ice-binding site of AFP III. ${ }^{30}$
In order to stimulate ice nucleation the LPIN will bind to a nano-ice crystal. Albeit opposite in the biological function - ice nucleating versus ice nucleating suppression - the LPIN and AFP face the same challenge in recognizing the surface of ice crystals. ${ }^{31}$ Both have developed strategies to preferentially bind ice crystal over liquid water. Our goal was to investigate whether they share the same mechanism which was postulated for AFP, i.e.: retardation of collective hydrogen bond dynamics over long distances. Here, we apply concentration dependent THz absorption spectroscopy to study the influence of the LPIN on the collective water network dynamics in order to unravel the molecular mechanism of the ice nucleation mechanism. As a test we will also investigate the effect of borate complexation - which similar to AFP shifts the hydration dynamics towards bulk water behavior. Special attention will be paid to the role of myo-inositol as an important binding motif for nano-ice crystals.

\section{Experimental section}

The LPIN was purified from $T$. trivittata larvae as described elsewhere. ${ }^{6}$ All other reagents were purchased from SigmaAldrich. The LPIN was analysed in TRIS buffer (25 mM TRIS, $50 \mathrm{mM} \mathrm{NaCl}, \mathrm{pH} 7.5)$ and in sodium tetraborate buffer $(25 \mathrm{mM}$ TRIS, $50 \mathrm{mM} \mathrm{NaCl}, 50 \mathrm{mM} \mathrm{Na}_{2} \mathrm{~B}_{4} \mathrm{O}_{7}, \mathrm{pH}$ 7.5).

In order to study the change in the fast collective water network dynamics, we used our p-Germanium ( $\mathrm{p}-\mathrm{Ge}$ ) laser difference spectrometer in a double beam configuration (see Fig. 1). ${ }^{32}$ We recorded the transmitted THz radiation in the frequency range from 2.4-2.7 THz depending on the sample concentrations in the sample cell. As a reference we measured the transmitted $\mathrm{THz}$ power when replacing the sample by buffer. We used Beer's law to calculate $\Delta \alpha$ as a function of solute concentration by subtracting the averaged absorption coefficients of the sample solution $\left(\alpha_{\text {sample }}\right)$ and of the buffer solution $\left(\alpha_{\text {reference }}\right)$ :

$$
\Delta \alpha=\alpha_{\text {sample }}-\alpha_{\text {reference }} .
$$

For each data point 60000-120000 pulses for both the sample and reference cells were recorded and averaged. All measurements were performed at low humidity $(<3 \%)$ and in a

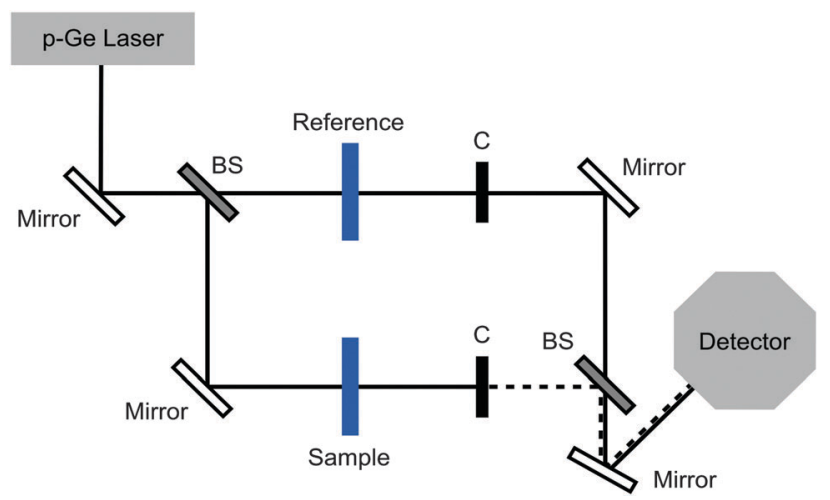

Fig. 1 Experimental set-up. Beam splitters (BSs) are used to set up two distinct beam paths. The transmission of the sample and of the reference cell is alternatively probed using two phase-shifted choppers (C). 
temperature stabilized cell at $5 \pm 0.1{ }^{\circ} \mathrm{C}$. The sample cell has dimensions of $1.5 \times 1.5 \mathrm{~cm}$ in the $x$ - and $y$-direction and $50 \mu \mathrm{m}$ in the $z$-direction, corresponding to the absorption length. The accurate spacing was determined using a Fourier transform infrared spectrometer, which recorded the interference between the transmitted and reflected waves (etalon structures). We used a beam splitter to set up two beam paths where the sample and the reference could be probed under identical conditions, in order to reduce systematic errors.

Circular dichroism (CD) spectra of the LPIN were recorded in the temperature range between 20 and $5{ }^{\circ} \mathrm{C}$ using a Jasco J-815 CD spectrometer (Jasco, Gross-Umstadt, Germany) using quartz cuvettes with a path length of $0.1 \mathrm{~cm}$. Spectra were recorded at wavelengths from $200-250 \mathrm{~nm}$. The spectra were baseline corrected. For each data point four spectra were averaged. The results are presented as mean residue ellipticity $[\Theta]$ in $\operatorname{deg} \mathrm{cm}^{2} \mathrm{dmol}^{-1}$ using a mean residue weight of $124.5 \mathrm{~g} \mathrm{~mol}^{-1}{ }^{6}$ The percentage of $\alpha$-helices and $\beta$-sheets in the two apolipoproteins of the LPIN was calculated using the web server K2D3. ${ }^{33}$

\section{Results and discussion}

Using CD spectroscopy we determined the secondary structure composition of the LPIN. The results are shown in Fig. 2. The lipoproteins contain 35\% $\beta$-sheets and $16 \% \alpha$-helices. No significant change in the secondary structure composition was observed between 5 and $20{ }^{\circ} \mathrm{C}$ (data not shown). It was reported that bacterial INPs from $P$. syringae contain an unusual $\beta$-helical fold ${ }^{12,16}$ which is also the major secondary structure motif in several insect AFPs. ${ }^{18,19}$ Consequently, Graether et al. suggested that AFPs and INPs share similar structural motifs. ${ }^{12}$ For the LPIN we found a high amount of $\beta$-structures, which gives room for speculation if the LPIN also partially consists of $\beta$-helical folds. However, the present data do not give evidence for this. CD and FTIR spectroscopy are incapable of confirming these structures with the required accuracy. ${ }^{34,35}$ Therefore, computational methods would be needed to further investigate the secondary structure motifs of the LPIN, and hence three dimensional structure information is needed.

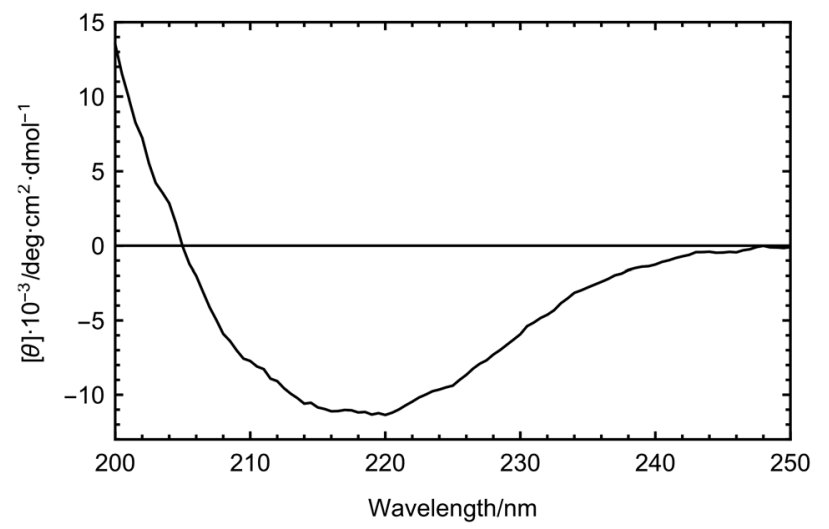

Fig. $2 \mathrm{CD}$ spectrum of the LPIN dissolved in $25 \mathrm{mM}$ TRIS buffer at $5{ }^{\circ} \mathrm{C}$.
In order to test the effect of the LPIN on the collective hydrogen bond dynamics we have carried out concentration dependent $\mathrm{THz}$ measurements. THz absorption spectroscopy is sensitive to protein-induced changes in the collective water dynamics. $^{23,32}$ The THz absorption of biomolecules, e.g. carbohydrates and proteins, in the 2.4 to $2.7 \mathrm{THz}$ range is in general smaller than the absorption of bulk water. Thus, in the absence of any influence of the biomolecule on the collective water network dynamics, we expect a linear decrease in THz absorption when increasing the sample concentration. ${ }^{21}$ However, for many biomolecules we found a nonlinear trend in the concentration dependence. ${ }^{23,24}$ Conversely, an initial increase in the $\mathrm{THz}$ absorption up to a characteristic protein concentration $c_{\text {max }}$ indicates that the protein is influencing the collective network motion in the vicinity of the protein. ${ }^{23}$ In a previous experimental and theoretical study we could show that an increase in $\mathrm{THz}$ absorption around $2.8 \mathrm{THz}$ is associated with a retardation in hydrogen bond dynamics. ${ }^{22}$ For AFP1 we found that the observation of a $\mathrm{THz}$ excess, i.e. a retardation of the hydrogen bond dynamics, is a necessary condition for antifreeze activity. ${ }^{22,23,32,36}$

The concentration dependent $\mathrm{THz}$ absorption coefficients in comparison to buffer for the LPIN at $5{ }^{\circ} \mathrm{C}$ are displayed in Fig. 3. The dashed line corresponds to the predicted $\mathrm{THz}$ absorption of a two-component mixture: the water is displaced by a $\mathrm{THz}$ transparent solute of the same size ( $\mathrm{THz}$ defect). Similar to all biological active AFPs we observed a small, but significant $\mathrm{THz}$ excess with a non-linear deviation from the predicted $\mathrm{THz}$ defect of up to $\sim 1.2 \mathrm{~cm}^{-1} \cdot{ }^{9,23-27}$ In order to test the correlation between the observed $\mathrm{THz}$ excess and biological activity we have added sodium borate which is known to inhibit ice nucleation. The addition of the inhibitor sodium borate resulted in a loss of the $\mathrm{THz}$ excess, revealing a correlation between ice nucleation activity and a measurable influence on the water network dynamics, similar to AFGP. ${ }^{9}$ Thus, we propose that also for the LPIN the influence on the collective

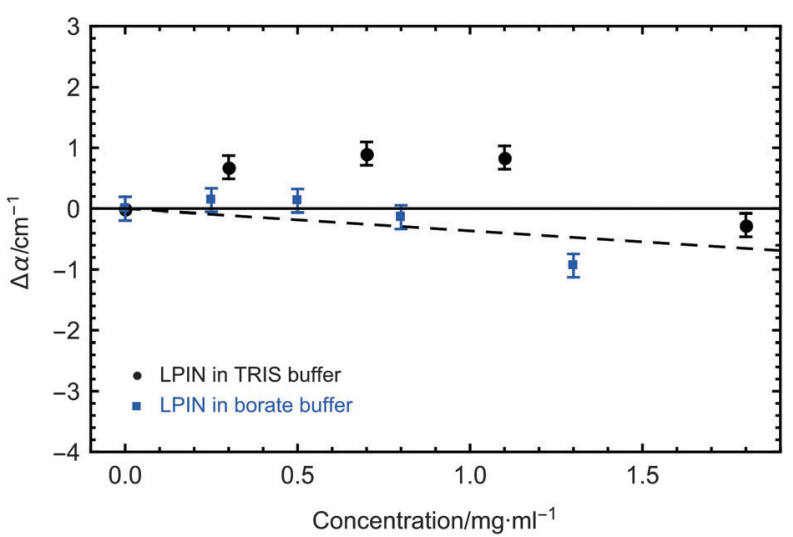

Fig. 3 Concentration-dependent $\mathrm{THz}$ absorption (averaged between 2.4 and $2.7 \mathrm{THz}$ ) of the LPIN dissolved in buffer relative to buffer. Measurements were carried out in $25 \mathrm{mM}$ TRIS buffer (black) and in $50 \mathrm{mM}$ borate buffer (blue) at $5{ }^{\circ} \mathrm{C}$. $2 \sigma$ standard deviations are reported as \pm SEM. The dashed line corresponds to the predicted THz absorption when subtracting water, which is displaced by the LPIN. 
water network dynamics is a necessary condition for ice nucleation activity, which at first glance is a surprising similarity between the LPIN and the AFGP. ${ }^{9}$

myo-Inositol as the functional head group of PI is a very uncommon component in insect lipoproteins. It is only present in insect lipoproteins which show ice nucleation activity and is known to play a crucial role in the ice nucleation activity of the LPIN. ${ }^{6}$ In order to test whether myo-inositol plays also an important role in the induced changes on the collective hydration bond dynamics we recorded the concentration dependent THz absorption at $5{ }^{\circ} \mathrm{C}$ displayed in Fig. 5. For myo-inositol, we observed a $\mathrm{THz}$ excess, i.e. an increase compared to the predicted $\mathrm{THz}$ defect, of up to $\sim 11 \mathrm{~cm}^{-1}$, indicating a strong myo-inositol-water interaction, which supports the idea that myo-inositol plays a crucial role in nucleation activity. This effect is temperature dependent, with a maximum $\mathrm{THz}$ effect at decreasing temperatures: additional measurements at higher temperatures showed a slightly less pronounced $\mathrm{THz}$ excess for myo-inositol (see Fig. 6, black data points).

Furthermore we compared the absorption of myo-inositol and the ice nucleation inhibitor sodium borate to a solution with sodium borate only. The results are shown in Fig. 5. The addition of sodium borate strongly reduced the $\mathrm{THz}$ absorption of myo-inositol, now following more closely the prediction for a solute that does not influence the water. Interestingly, upon addition of the ice nucleation inhibitor sodium borate we observed even a decrease of $\mathrm{THz}$ absorption below the predicted $\mathrm{THz}$ defect. At first glance this unexpected result can be explained by the fact that the reference sample, the solvated

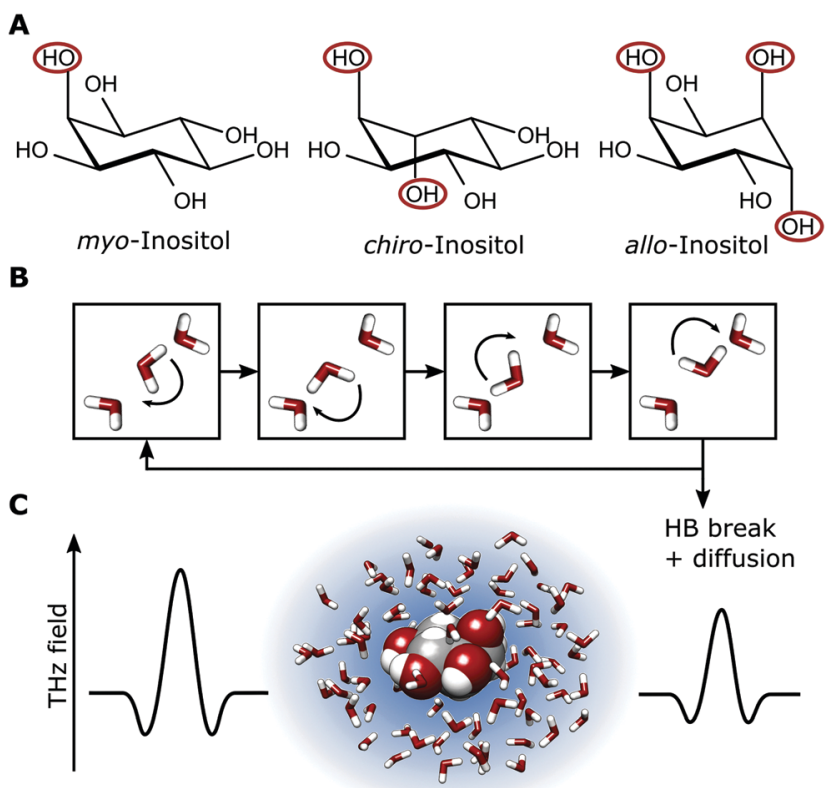

Fig. 4 (A) Conformation of the three investigated inositol stereoisomers The axial $\mathrm{OH}$ groups are highlighted. (B) Illustration of the underlying $\mathrm{H}$-bond rearrangements in the water network. (C) Representation of hydrated myo-inositol, as probed by $\mathrm{THz}$ absorption spectroscopy. The blue shadow schematically represents the degree of retardation of the collective $\mathrm{H}$-bond network dynamics in the hydration shell.

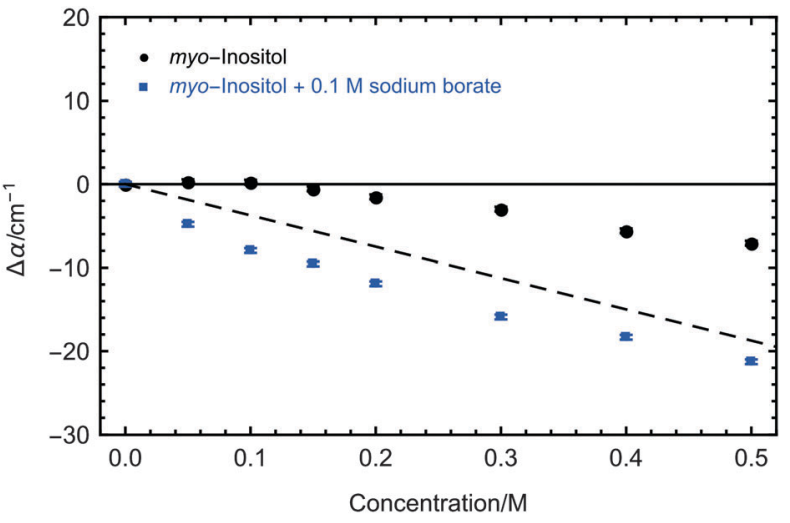

Fig. 5 Concentration-dependent THz absorption (averaged between 2.4 and $2.7 \mathrm{THz}$ ) of $m y o$-inositol dissolved in water relative to water (black) and of myo-inositol dissolved in $0.1 \mathrm{M}$ sodium borate solution relative to $0.1 \mathrm{M}$ sodium borate solution (blue). Measurements were carried out at $5{ }^{\circ} \mathrm{C} .2 \sigma$ standard deviations are reported as \pm SEM. The dashed line corresponds to the predicted $\mathrm{THz}$ absorption when subtracting water, which is displaced by inositol.

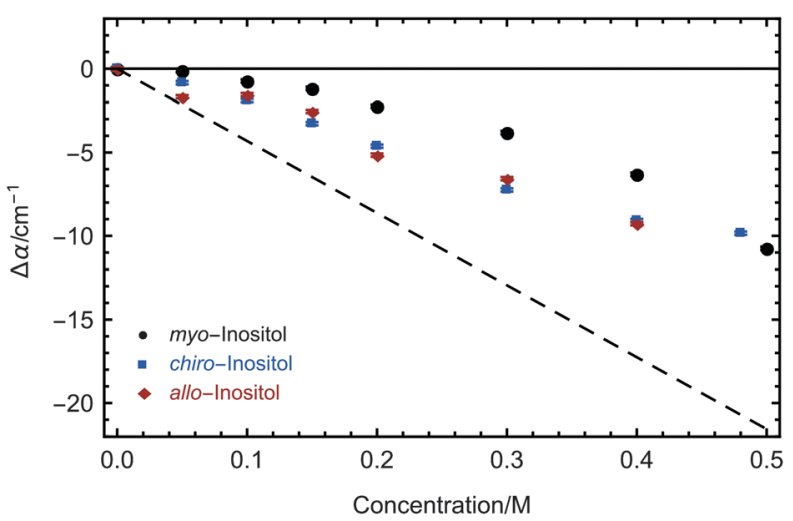

Fig. 6 Concentration-dependent $\mathrm{THz}$ absorption (averaged between 2.4 and $2.7 \mathrm{THz}$ ) of myo-, chiro- and allo-inositol dissolved in water relative to water (black, blue and red). Measurements were carried out at $20{ }^{\circ} \mathrm{C} .2 \sigma$ standard deviations are reported as \pm SEM. The dashed line corresponds to the predicted $\mathrm{THz}$ absorption when subtracting water, which is displaced by inositol.

negatively charged borate ion, is expected to show characteristic rattling modes in the investigated frequency range. ${ }^{37}$ Upon binding of the negatively charged borate ion to the $\mathrm{OH}$ groups of myo-inositol these rattling modes should disappear due to desolvation. We attribute the overall negative $\Delta \alpha$ of solvated myo-inositol complexed with borate compared to solvated borate to a disappearance of the rattling modes and a diminishing effect on the myo-inositol-water interaction. It is most likely that borate ions show the same effect when myo-inositol as the head group of PI is located on the surface of the LPIN, thereby inhibiting ice nucleation.

Our investigations of AFP1 and DAFP revealed the importance of an exact positioning of a rigid helical structure that positions threonine residues accurately for ice-binding for biological functions, as was tested by choosing distinct mutants of AFP1. ${ }^{36}$ Therefore we have investigated three different inositol 
stereoisomers (myo-, chiro- and allo-inositol, see Fig. 4) to address the question, whether the specific positions of the $\mathrm{OH}$ groups in the inositol stereoisomers are of importance for the influence on the water network dynamics. The concentration dependent THz absorption of myo-inositol was compared to those of chiro- and allo-inositol in order to explore the importance of the configuration of the six $\mathrm{OH}$ groups in the influence on the water network dynamics. Fig. 6 shows the difference in $\mathrm{THz}$ absorption $(\Delta \alpha)$ of all three inositol stereoisomers compared to water recorded at $20{ }^{\circ} \mathrm{C}$. myo-Inositol has one out of six $\mathrm{OH}$ groups in the axial position, and chiro-inositol shows two and allo-inositol even three axial $\mathrm{OH}$ groups. None of later two has been found in INPs. Although we observed a pronounced $\mathrm{THz}$ excess for all stereoisomers, the THz excess of myo-inositol exceeds the excess of other stereoisomers, revealing a more significant myo-inositol-water interaction compared to chiro- and allo-inositol. Our results suggest that the specific and precise arrangement of the $\mathrm{OH}$ groups in inositol molecules is indeed an important factor determining the strength of the interaction with the surrounding water network (similar to that found for AFP1). We speculate that the effective interaction of myo-inositol plays an important role in the biological activity of the LPIN, with the precise arrangement of the $\mathrm{OH}$ groups determining the actual ice nucleation strength as tested by the variation of the positioning.

Our measurements indicate that the LPIN uses a similar two tier molecular mechanism for the preferred binding to nanoice crystals as do AFPs: these proteins influence the collective hydration bond network dynamics and exhibit a local mechanism in which the exact positioning of the $\mathrm{OH}$ groups plays a decisive role. ${ }^{9,23,38}$

\section{Conclusions}

In summary, the strong myo-inositol-water interaction seems to be a crucial feature for ice nucleation activity of LPINs. Our results indicate that the precisely arranged $\mathrm{OH}$ groups of the myo-inositol head of PI represent part of the ice-binding site of the LPIN, similar to the threonine residues in several AFPs. Furthermore, we found a small but significant influence of the LPIN on the collective water network dynamics, which is partly attributed to the interaction of $m y o$-inositol with water. Sodium borate diminishes the influence on the water network in both cases. The loss of the $\mathrm{THz}$ excess correlates with a loss of ice nucleation activity in the case of LPINs. ${ }^{6}$

For AFP, we speculated that the formation of a hydration funnel, i.e. a gradient of retardation in hydrogen bond dynamics towards the ice-binding site, leads to a reduction of the entropic cost for binding as part of the molecular binding mechanism. Although both protein classes serve opposite biological functions, a similar molecular mechanism seems to be responsible for the ice nucleation activity of INPs.

\section{Conflict of interest}

The authors declare no competing financial interest.

\section{Acknowledgements}

The authors thank E. E. Haller and E. Bründermann for the supply of the Ge laser and Ge detector crystals, ${ }^{39}$ Konrad Meister for fruitful discussions and Hendrik Vondracek for technical support. This work is supported by the Cluster of Excellence RESOLV (EXC 1069) funded by the Deutsche Forschungsgemeinschaft.

\section{References}

1 H. Kawahara, Psychrophiles: from Biodiversity to Biotechnology, Springer, Heidelberg, 2008, pp. 229-246.

2 K. E. Zachariassen and E. Kristiansen, Cryobiology, 2000, 41, 257-279.

3 J. G. Duman, Annu. Rev. Physiol., 2001, 63, 327-357.

4 E. B. Moore and V. Molinero, Nature, 2011, 479, 506-508.

5 J. G. Duman, L. G. Neven, J. M. Beals, K. R. Olson and F. J. Castellino, J. Insect Physiol., 1985, 31, 1-8.

6 L. G. Neven, J. G. Duman, M. G. Low, L. C. Sehl and F. J. Castellino, J. Comp. Physiol., B, 1989, 159, 71-82.

7 K. L. Yeung, E. E. Wolfa and J. G. Duman, J. Vac. Sci. Technol., B: Microelectron. Nanometer Struct.-Process., Meas., Phenom., 1991, 9, 1197-1201.

8 J. G. Duman, D. Wen Wu, P. K. Wolber, G. M. Mueller and L. G. Neven, Comp. Biochem. Physiol., Part B: Biochem. Mol. Biol., 1991, 99, 599-607.

9 S. Ebbinghaus, K. Meister, B. Born, A. L. DeVries, M. Gruebele and M. Havenith, J. Am. Chem. Soc., 2010, 132, 12210-12211.

10 L. R. Maki, E. L. Galyan, M.-M. Chang-Chien and D. R. Caldwell, Appl. Microbiol., 1974, 28, 456-459.

11 L. M. Kozloff, M. A. Schofield and M. Lute, J. Bacteriol., 1983, 153, 222-231.

12 S. P. Graether and Z. Jia, Biophys. J., 2001, 80, 1169-1173.

13 H. Kawahara, J. Biosci. Bioeng., 2002, 94, 492-496.

14 J. S. H. Lorv, D. R. Rose and B. R. Glick, Scientifica, 2014, 2014, 20.

15 J. A. Raymond and A. L. DeVries, Proc. Natl. Acad. Sci. U. S. A., 1977, 74, 2589-2593.

16 C. P. Garnham, R. L. Campbell, V. K. Walker and P. L. Davies, BMC Struct. Biol., 2011, 11, 36.

17 C. P. Garnham, R. L. Campbell and P. L. Davies, Proc. Natl. Acad. Sci. U. S. A., 2011, 108, 7363-7367.

18 S. P. Graether, M. J. Kuiper, V. K. Walker, Z. Jia, B. D. Sykes and P. L. Davies, Nature, 2000, 406, 325-328.

19 Y.-C. Liou, A. Tocilj, P. L. Davies and Z. Jia, Nature, 2000, 406, 322-324.

20 U. Heugen, G. Schwaab, E. Bründermann, M. Heyden, X. Yu, D. M. Leitner and M. Havenith, Proc. Natl. Acad. Sci. U. S. A., 2006, 103, 12301-12306.

21 D. M. Leitner, M. Gruebele and M. Havenith, HFSP J., 2008, 2, 314-323.

22 M. Heyden and M. Havenith, Methods, 2010, 52, 74-83.

23 S. Ebbinghaus, S. J. Kim, M. Heyden, X. Yu, U. Heugen, M. Gruebele, D. M. Leitner and M. Havenith, Proc. Natl. Acad. Sci. U. S. A., 2007, 104, 20749-20752. 
24 B. Born, S. J. Kim, S. Ebbinghaus, M. Gruebele and M. Havenith, Faraday Discuss., 2009, 141, 161-173.

25 K. Meister, S. Ebbinghaus, Y. Xu, J. G. Duman, A. L. DeVries, M. Gruebele, D. M. Leitner and M. Havenith, Proc. Natl. Acad. Sci. U. S. A., 2013, 110, 1617-1622.

26 K. Meister, J. G. Duman, Y. Xu, A. L. DeVries, D. M. Leitner and M. Havenith, J. Phys. Chem. B, 2014, 118, 7920-7924.

27 Y. Xu, A. Bäumer, K. Meister, C. Bischak, A. L. DeVries, D. M. Leitner and M. Havenith, Chem. Phys. Lett., 2016, 647, 1-6.

28 V. Conti Nibali and M. Havenith, J. Am. Chem. Soc., 2014, 136, 12800-12807.

29 Y. Xu and M. Havenith, J. Chem. Phys., 2015, 143, 170901.

30 K. Meister, S. Strazdaite, A. L. DeVries, S. Lotze, L. L. C. Olijve, I. K. Voets and H. J. Bakker, Proc. Natl. Acad. Sci. U. S. A., 2014, 111, 17732-17736.

31 K. A. Sharp, Proc. Natl. Acad. Sci. U. S. A., 2011, 108, 7281-7282.
32 A. Bergner, U. Heugen, E. Bründermann, G. Schwaab, M. Havenith, D. R. Chamberlin and E. E. Haller, Rev. Sci. Instrum., 2005, 76, 063110-063115.

33 C. Louis-Jeune, M. A. Andrade-Navarro and C. PerezIratxeta, Proteins: Struct., Funct., Bioinf., 2011, 80, 374-381.

34 V. Sieber, F. Jurnak and G. R. Moe, Proteins: Struct., Funct., Genet., 1995, 23, 32-37.

35 R. Khurana and A. L. Fink, Biomater. Sci., 2000, 78, 994-1000.

36 S. Ebbinghaus, K. Meister, M. B. Prigozhin, A. L. DeVries, M. Havenith, J. Dzubiella and M. Gruebele, Biophys. J., 2012, 103, L20-L22.

37 D. A. Schmidt, S. Funkner, B. Born, R. Gnanasekaran, G. Schwaab, D. M. Leitner and M. Havenith, J. Am. Chem. Soc., 2009, 131, 18512-18517.

38 T. Q. Luong, P. K. Verma, R. K. Mitra and M. Havenith, Biophys. J., 2011, 101, 925-933.

39 E. Bründermann, A. M. Linhart, L. Reichertz, H. P. Röser, O. D. Dubon, W. L. Hansen, G. Sirmain and E. E. Haller, Appl. Phys. Lett., 1996, 68, 3075-3077. 